\title{
« La méthode orale » à l'Institut français d'Athènes : innovations pédagogiques dans l'enseignement du français en Grèce (1946-1961)
}

Loukia Efthymiou et Nicolas Manitakis

\section{OpenEdition \\ Journals}

\section{Édition électronique}

URL : https://journals.openedition.org/dhfles/4456

DOI : $10.4000 /$ dhfles.4456

ISSN : 2221-4038

Éditeur

Société Internationale pour l'Histoire du Français Langue Étrangère ou Seconde

Édition imprimée

Date de publication : 1 décembre 2017

Pagination : 121-137

ISSN : 0992-7654

Référence électronique

Loukia Efthymiou et Nicolas Manitakis, « « La méthode orale » à l'Institut français d'Athènes :

innovations pédagogiques dans l'enseignement du français en Grèce (1946-1961) », Documents pour I'histoire du français langue étrangère ou seconde [En ligne], 58-59 | 2017, mis en ligne le 04 juin 2018, consulté le 25 mars 2023. URL : http://journals.openedition.org/dhfles/4456 ; DOl : https://doi.org/ $10.4000 /$ dhfles.4456

Ce document a été généré automatiquement le 25 mars 2023.

Tous droits réservés 


\title{
« La méthode orale » à l'Institut français d'Athènes : innovations pédagogiques dans l'enseignement du français en Grèce (1946-1961)
}

\author{
Loukia Efthymiou et Nicolas Manitakis
}

1 Lors d'une conférence tenue en 1950 au Centre International d'Études Pédagogiques de Sèvres, l'Inspecteur général Pierre Clarac faisait l'éloge et érigeait au rang d'exemple la «méthode orale » appliquée par le personnel de l'Institut Français d'Athènes après la guerre ${ }^{1}$. Clarac, qui était également membre du jury du Diplôme de professeur de français à l'étranger de l'École de la Sorbonne, était récemment rentré d'une visite d'inspection en Grèce. Son témoignage enthousiaste et l'intérêt qu'il suscita parmi une assistance composée de professeurs de langues vivantes suggère que la méthode mise en vigueur à Athènes par le directeur de l'établissement culturel français, Octave Merlier, fit alors figure d'innovation pédagogique (Voutsinas 1951).

2 C'est que, tout d'abord, cette technique d'enseignement déjà vieille de près d'un demisiècle en Europe, s'inscrivant dans un vaste projet éducatif admirablement orchestré par Merlier, donna naissance à des pratiques de classe inédites jusque-là, à notre connaissance, en Grèce. Qui plus est, remodelée minutieusement et appliquée avec soin et "rigueur $»^{2}$, elle apparut également en France - dans un contexte d'initiatives d'aménagement linguistique et didactique en matière de FLE - comme une stratégie d'apprentissage intéressante méritant largement une diffusion en dehors de l'espace grec. Par le biais donc de la reconstitution de l'histoire d'une expérience pédagogique franco-hellénique qui dura quinze ans (1946-1961), la présente étude se propose de mettre en valeur un exemple à la fois de transfert d'une méthode enseignante et d'ajustement de ses procédés et supports pédagogiques au sein de différentes institutions d'accueil. 


\section{La gestation du projet (1925-1945)}

3 Fondé au début du $\mathrm{XX}^{\mathrm{e}}$ siècle en tant qu'annexe pédagogique de l'École française d'Athènes, l'Institut supérieur d'Études françaises, renommé après 1945 Institut français d'Athènes, est vite devenu l'un des principaux centres d'enseignement de la langue française dans la capitale grecque. Son développement fulgurant à partir de l'entredeux-guerres fut nottament l'œuvre d'Octave Merlier, professeur de français et néohelléniste très attaché à la Grèce.

Dès son arrivée à Athènes, Merlier avait constaté non seulement l'inefficacité de la méthode traditionnelle qui était employée dans la classe de français par des maîtres grecs recrutés souvent, par surcroît, sans formation initiale ${ }^{1}$, mais aussi l'inadéquation des «méthodes ordinaires de la métropole » utilisées par les professeurs de l'Institut (instituteurs, licenciés ou agrégés de lettres et de grammaire) pour enseigner le français à un public d'apprenants étrangers. Centrées en priorité sur l'étude de la grammaire et de la littérature, ces pratiques méthodologiques délaissaient l'enseignement oral, de sorte que les élèves grecs, même ceux qui réussissaient à "acquérir des connaissances théoriques", ne parvenaient point à s'exprimer en français².

5 Malgré la gravité de la question, peu d'initiatives furent engagées avant la guerre pour pallier ces déficiences. On note en particulier, en 1936, la publication aux éditions Le Progrès d'une Méthode de Prononciation Française à l'usage des élèves grecs, bel exemple d'ouvrage pédagogique tenant compte des spécificités linguistiques de la population hellène ${ }^{3}$. Son auteure : une institutrice française, Mlle Renvoysé, recrutée en 1928 pour enseigner le français aux élèves débutants - très peu nombreux encore, il est vrai, parmi les effectifs de l'Institut.

6 C'est sous l'effet des changements opérés au sein de la population étudiante de l'établissement dès les dernières années de la guerre et accentués à la Libération avec le rattachement des cours de l'Alliance Française transformés en Écoles Annexes, que la question de la pertinence des méthodes d'enseignement utilisées dans ses classes commença à se poser avec acuité. Concrètement, au cours de cette période, non seulement ses effectifs grimpèrent en flèche mais aussi l'importance du nombre des débutants de moins de seize ans y progressa considérablement. D'où la nécessité, d'une part, de privilégier l'aspect oral de l'enseignement afin de satisfaire les besoins éducatifs de la nouvelle clientèle et, d'autre part, de renforcer l'effectif du personnel enseignant.

7 Dans un premier temps, l'établissement français ouvrit, en 1943, un Institut annexe, pour recueillir le trop-plein du bâtiment central. Puis, pour la première fois depuis sa création, il procéda, en deux phases, au recrutement de professeurs hellènes, diplômés pour la plupart de son Cours Spécial - structure de formation initiale des francisants reconnue depuis 1930 par l'État grec.

8 La première tranche d'enseignants grecs fut embauchée en pleine Occupation allemande. Réunis - essentiellement depuis 1944 - à l'Institut annexe, ils se virent confier les élèves de moins de 16 ans. Cette assignation devrait s'avérer déterminante pour l'avenir de la méthode traditionnelle au sein de l'Institut. En effet, elle fut vite rejetée par ces nouveaux professeurs, car taxée d'inadéquation pour un public de jeunes apprenants de plus en plus nombreux. Il était désormais question de la 
remplacer au plus vite. L'enjeu était de taille. Faisant preuve d'un esprit d'expérimentation et d'innovation exceptionnel, cette première équipe, composée d'Hélène Papanicolaou, Hellé Papastamatiou, Irène Tsouchlou, Hellé Tzanetaki, Georges et Hélène Mourelos, réussit à transformer l'annexe de la rue Sina en un véritable laboratoire de pédagogie appliquée par la mise en chantier de pratiques d'enseignement radicalement nouvelles en Grèce ${ }^{4}$.

9 À la Libération, l'établissement culturel français fit une seconde fois appel à ses anciens diplômés pour pourvoir les très nombreux postes vacants dans les Écoles Annexes qu'il dirigeait depuis peu. Or, pour réussir l'intégration des nouveaux professeurs - de sexe féminin dans leur écrasante majorité - dans les Écoles annexes, il fallait à brève échéance les former aux méthodes d'enseignement qu'on s'apprêtait à y mettre en application. L'équipe dirigeante se trouvait dès lors confrontée à un double défi.

\section{Faire œuvre pédagogique}

\section{La « méthode orale »}

En quoi consistait concrètement la méthode mise au point à Athènes en 1946 ? Certes, elle était largement inspirée par la méthode directe appliquée, au début du XXe siècle, en France dans l'enseignement des langues vivantes. Merlier, lui-même, le reconnaissait d'ailleurs, quand il affirmait que « l'idée [était] ancienne » ${ }^{1}$. Il n'empêche qu'en Grèce, c'est l'Institut qui, selon les sources consultées, fut le seul établissement éducatif à l'avoir systématiquement mise en œuvre² ${ }^{2}$.

11 Appelée par ses promoteurs athéniens "méthode orale ", elle visait à créer dans la classe de débutants les conditions d'acquisition de la langue maternelle, afin de déclencher chez des apprenants de tout âge « des automatismes verbaux stables [...], et [de] ne permettre la formation d'automatismes visuels que lorsque les premiers seront solidement constitués». Il s'ensuit que la stratégie d'apprentissage en question conçue selon un ordre rigoureux dans sa progression (du concret à l'abstrait, du fréquent au moins fréquent, du simple au complexe) -, préconisait un enseignement d'un an, puis, à partir de 1947, de deux ans, sans livres. Elle s'appuyait dès lors essentiellement sur l'usage de l'image, du « jeu » et de la chanson et accordait « une place très importante à la prononciation ». La matière orale devait être toujours groupée autour de centres d'intérêt ("vie quotidienne", "maison", "école", "métiers», etc.) et répartie en exercices de prononciation, de vocabulaire et de "grammaire concrète " ${ }^{3}$. À la fin de la deuxième année un bagage d'environ 1500 mots et expressions d'usage courant devait être acquis par les enfants.

Dans le cadre d'un enseignement ainsi conçu par les promoteurs de la version grecque de la méthode directe, le matériel d'appoint était censé jouer un rôle crucial. C'est notamment Hélène Metaxas-Mourelos qui se distingua dans ce domaine : à la tête d'une équipe, elle mit sur pied, dès 1946, des aides audio-visuelles destinées à l'enseignement du vocabulaire. Ce matériel iconographique fut par la suite reproduit et diffusé dans les annexes de l'Institut grâce au service d'édition-imprimerie mis en place en $1949^{4}$. Comme l'attestent des photos de l'époque, toutes les salles des Écoles Annexes furent équipées de tableaux muraux et les élèves furent invités à se procurer des cartes illustrées fabriquées par l'équipe pédagogique grecque. 
13 La « révolution » méthodologique introduite à l'Institut après la guerre ne connut pas des débuts faciles toutefois. Quand, à l'instigation du directeur, la méthode orale fut mise à l'essai pendant une année dans une seule classe, elle suscita des réactions plurielles: le scepticisme des professeurs de l'établissement "que l'idée d'un travail sans livres et cahiers effrayait ", la résistance et les objections des élèves et de leurs parents qui se méfiaient surtout de son aspect ludique. "Les élèves, se rappelait en 1955 Merlier, croyaient indigne d'eux de ne posséder ni livre ni cahier; les parents ne comprenaient pas qu'on pût enseigner sans un livre et un cahier ». Ils n'y «voyaient, surenchérissaient en 1957 ses collaboratrices les plus proches, qu'un amusement et une perte de temps pour leurs enfants ». Mais c'est justement le caractère "agréable » d'une méthode, du reste appuyée sur des principes solides, qui constitua, selon ces mêmes professeures, l'un de ses principaux avantages : «Tous les ans, signalent-elles, nous sommes heureux de constater l'atmosphère joyeuse qui transforme nos leçons en parties de plaisir pour nos élèves. Ainsi le premier contact avec la langue étrangère, d'ordinaire si ardu et monotone, s'accomplit dans la joie $»^{5}$.

\section{Former les professeurs de français à la nouvelle méthode}

14 Une fois les oppositions au sein de l'Institut apaisées à la suite des « résultats remarquables » obtenus " en sept mois de travail $»^{6}$, la formation pédagogique des professeurs, futurs et en exercice, à la nouvelle pratique enseignante préoccupa sérieusement Merlier et ses collaborateurs. Conscients de l'importance de cet enjeu pour la réussite de la méthode, ils développèrent leur action sur trois plans.

En premier lieu, ils initièrent, dès 1946-1947, tous les professeurs des Écoles Annexes au programme d'enseignement oral par le biais de "congrès" et de séances de perfectionnement. Parallèlement, ils chargèrent l'équipe pédagogique travaillant sur la méthode orale d'élaborer des manuels destinés aux maîtres. Dès 1948, un ouvrage intitulé Livre du professeur pour l'enseignement oral fut préparé par Hélène Papanicolaou et Irène Tsouchlou avec l'aide d'Hélène Metaxas-Mourelos, chargée du choix des chansons. Ce matériel, ronéotypé à 300 exemplaires et distribué aux professeurs de l'Institut, contenait tout un programme détaillé établissant la progression à suivre au «Cycle oral ». Enfin, la direction de l'Institut s'employa à former à la nouvelle méthode les futurs francisants dans le cadre du Cours Spécial. Cette préparation fut, d'ailleurs, ouverte également aux professeurs des annexes de l'Institut et «des lycées d'Athènes $»^{7}$.

16 À l'état embryonnaire jusqu'à la guerre, le stage pédagogique des élèves du Cours de préparation au professorat de français fut doté, en 1948, d'une organisation complète qui, " inspirée de la tradition universitaire française", comportait une double initiation: théorique et pratique (Efthymiou 2015: 97-99). À partir de 1950 notamment, la formation théorique, confiée à l'un des initiateurs de la méthode orale à l'Institut, le futur professeur de philosophie Georges Mourelos ${ }^{8}$, fut illustrée par des «travaux d'outillage pédagogique » qui visaient à entraîner « les étudiants » du Cours Spécial à la confection du matériel scolaire (tableaux, dessins, objets de carton, poupées, marionnettes, etc.) destiné à concrétiser l'enseignement direct de la langue dans les cours oraux pour jeunes débutants 9 . Les futurs professeurs apprenaient également comment insérer "le programme des fêtes scolaires dans l'enseignement de l'année ", comment diriger les jeux éducatifs et travailler en classe avec la chanson, afin de 
développer les compétences orales de leurs élèves. À cet effet, un cours hebdomadaire de lecture musicale et de solfège fut introduit dans leur formation à partir de 1948. À noter, enfin, un « cours de conversation courante par petits groupes » dispensé à partir de $1956^{10}$.

L'initiation pratique au « métier de professeur » fut par ailleurs organisée de manière méticuleuse par les responsables de l'Institut. Étalée sur les deux années du cursus, elle était passive et active à la fois. Le but était de "familiariser [les étudiants] avec l'application de la 'méthode orale' «" ${ }^{11}$. Concrètement, après avoir assisté à raison d'une semaine complète aux classes-modèles de l'Institut Annexe pour prendre contact avec la méthode pratiquée, les stagiaires donnaient un enseignement de 8 à 18 heures dans le Cycle Élémentaire. Leurs propres leçons préparées sur les indications des directrices de stage, puis corrigées, critiquées et commentées, reproduisaient fidèlement les leçons-modèles élaborées lors de longues séances de travail animées de causeries techniques ${ }^{12}$. Enfin, un rapport détaillé sur l'ensemble du stage évaluait la prononciation, la connaissance de la langue concrète et les dispositions pédagogiques des futurs professeurs.

La mise sur pied de manière coordonnée de cet édifice pédagogique complexe dans les années 1950 permit au grand projet de Merlier de prendre consistance: créer à l'Institut un « centre pédagogique » dont la mission se partagerait entre enseignement, formation et élaboration de méthodes d'apprentissage. Les deux premières assignations avaient été depuis longtemps reconnues ou réservées à l'établissement français par l'État hellénique. Il lui restait encore à s'imposer officiellement comme autorité dans le domaine de la méthodologie des langues vivantes, voire à être reconnu comme une instance apte à planifier des réformes éducatives et à les mettre en œuvre dans les établissements publics grecs ${ }^{13}$.

\section{Faire œuvre de diffusion}

Or, la réalisation de ce projet de grande envergure, qui se résumait en substance à l'implantation de la méthode orale dans d'autres structures scolaires, reposait dans une large mesure sur la planification de stratégies efficaces de diffusion des résultats du travail fait à l'Institut dans ce domaine. Merlier opéra notamment sur deux fronts. D'un côté, il mit en place en Grèce des mécanismes de promotion de l'œuvre pédagogique accomplie. De l'autre, il assigna à ses collaborateurs une mission de médiation: à savoir, de faire connaître cette expérience non seulement dans les milieux enseignants grecs mais aussi en dehors de l'espace hellénique.

Dès 1946, Merlier conçut à cet effet une institution importante: les «congrès de l'enseignement du français », destinés aux professeurs en herbe ou en exercice dans tous les types d'établissements scolaires. Quatre rencontres pédagogiques, vite revêtues d'un caractère solennel en raison de la présence des autorités helléniques et françaises (ambassadeurs, ministres, hauts fonctionnaires), furent tenues entre 1947 et 1955 au bâtiment central d'Athènes lors des vacances de Pâques (1947, 1948, 1950 et 1955). Elles furent par ailleurs relayées en province par des démonstrations de leçons-modèles organisées dans les Écoles annexes de l'établissement. Toutes ces manifestations pédagogiques permirent de présenter à la communauté enseignante, entre autres, les principes théoriques de l'enseignement oral et de démontrer son efficacité ${ }^{1}$; de formuler, aussi, des suggestions et des vœux pour l'avenir dont le plus important fut la 
mise en application de la nouvelle méthode dans les établissements publics et libres grâce au concours des professeurs de l'Institut.

21 Mais la campagne de Merlier en faveur de l'adoption officielle de la nouvelle pratique enseignante resta finalement sans fruits, malgré tous les procédés mis en œuvre à cet effet; malgré également l'appui que certains hauts fonctionnaires grecs du ministère de l'Éducation nationale lui avait apporté. La Direction de l'enseignement secondaire refusa fermement, tout au long des années 1950, d'y donner son aval sous prétexte de son inapplicabilité en milieu scolaire grec ${ }^{2}$.

Les échecs essuyés sur le front intérieur furent, dans une certaine mesure, compensés par l'expérience heureuse de l'implantation de la méthode à l'étranger. C'est notamment par le biais d'un ancien professeur de l'Institut, détaché en Grèce entre 1947 et 1950, qu'un tel transfert put aboutir. Au cours de son séjour athénien, Francis Baulier, agrégé de grammaire avait été gagné à la méthode orale et s'était progressivement spécialisé dans l'enseignement de la phonétique. Nommé en 1950 proviseur au lycée français de Vienne, il entreprit, à partir de 1951-1952, de transposer et d'adapter, « ingénieusement » selon Milliex, cette technique d'enseignement dans un nouveau cadre institutionnel. Dans la lettre-hommage qu'il envoya à son ancien directeur en 1952, il fit état du succès de la méthode "préparée en Grèce » dans son lycée autrichien: résultats pédagogiques très encourageants - sinon brillants -, augmentation spectaculaire des effectifs - et ceci malgré les heurts produits et les difficultés rencontrées ${ }^{3}$.

23 Au-delà de ce cas de « dissémination » réussie, l'équipe pédagogique de l'Institut, sous l'impulsion de son directeur, essaya de faire connaître à l'échelle européenne la "méthode orale ", voire de l'ériger en exemple. Son travail fut d'abord présenté en 1953 au Congrès International des Professeurs de Langues vivantes organisé par le Centre International d'Études Pédagogiques à Sèvres (Voutsinas 1953). L'intérêt que cette intervention suscita, poussa probablement les responsables des Cahiers Pédagogiques à solliciter, en 1956, sa contribution à un numéro consacré au «français langue étrangère ${ }^{4}$. Déjà, l'invitation de la part d'une revue spécialisée dans la recherche pédagogique atteste l'infiltration de l'équipe grecque dans des réseaux producteurs de réflexion méthodologique innovante ${ }^{5}$; le poids, toutefois, de sa présence dans ce numéro spécial par le biais de la publication de trois articles rédigés par six professeurs est, en outre, une belle preuve de reconnaissance de sa compétence scientifique en la matière (Papanicolaou, Papastamatiou, Tsouchlou, Tzanetaki 1957 : 28-30 ; Burney 1957 : 31-32 ; Efthymiatou 1957 : 61-62).

24 Enfin, Merlier chercha à promouvoir l'œuvre enseignante effectuée par ses professeurs en l'associant aux recherches menées à l'École normale supérieure de Saint-Cloud en vue de l'élaboration du Français élémentaire. Selon le directeur, le succès de la méthode orale appliquée à l'Institut athénien pratiquement depuis la Libération ne pouvait qu'avoir servi d'inspiration à ce vaste projet de politique linguistique française lancé en 1951 par le ministre de l'Éducation nationale André Marie sous l'impulsion de l'UNESCO'.

25 Un grand nombre d'indices corroborent cette assertion de Merlier. Si, à l'exemple du Basic English, le «centre d'étude du Français élémentaire» remit en 1954 les listes retenues pour constituer un «français réduit» (1475 «mots lexicaux et grammaticaux ») employé de façon usuelle en toutes circonstances et fit paraître, en 1956, une première édition du français élémentaire ${ }^{7}$, les «Rapports sur l'activité de 
l'Institut » révèlent que les promoteurs de l'enseignement oral travaillaient depuis 1947 déjà avec l'idée d'un français de base : « Nos débutants, écrivait Merlier, [...] manient, à la fin d'une deuxième année de Cours Oral, près d'un millier de mots d'usage quotidien $»^{8}$. En 1948, au congrès d'avril, le théoricien de la méthode, Georges Mourelos, présentait la liste détaillée des mots enseignés au Cours Oral : en tout « 750 termes " au moins. Ce "vocabulaire type " de la première année du Cours Oral était composé de noms et d'adjectifs (366), de noms composés (41), de verbes (91), de motsoutils (73), d'expressions usuelles (90), du vocabulaire des parties du corps et du vocabulaire des chansons ${ }^{9}$. Tout cela laisse ainsi penser que des pratiques pédagogiques innovantes, telle la constitution d'un vocabulaire limité de mots courants en français, avaient déjà été expérimentées par les enseignants de l'Institut athénien bien avant qu'elles ne soient mises en œuvre en France. De fait, en 1950, l'inspecteur général Clarac découvrait tout le travail méthodologique accompli à l'Institut et s'en félicitait. En décembre 1951, enfin, ce fut au tour du ministre André Marie de s'en informer lors de sa visite officielle en Grèce. Il était accompagné du président de la Commission chargée de suivre de près les enquêtes menées sur la version française du Basic English, Marcel Abraham, directeur également du Service Universitaire des Relations avec l'Étranger et ami de longue date de Merlier.

Quel bilan tirer, de la campagne de diffusion de la " méthode orale » réalisée entre 1946 et 1961 par les responsables de l'Institut français d'Athènes? Par le biais de l'organisation de rencontres pédagogiques et de leçons-modèles, l'Institut réussit, sans aucun doute, à faire connaître la nouvelle méthode non seulement parmi ses professeurs, mais aussi dans d'autres milieux d'enseignants grecs de la langue française, et cela tant dans la région de la capitale grecque, qu'en province. Fait inespére, dépassant largement les prévisions de ses promoteurs, l'innovation méthodologique de l'institution athénienne fut "exportée» en Autriche et, par ricochet, atteignit même la France, d'où elle tenait son origine.

\section{En guise de conclusion : le poids d'un héritage}

Tout compte fait, la «méthode orale » minutieusement mise au point à l'Institut français d'Athènes fut considérée dans l'après-guerre en Grèce comme une innovation intéressante dans la mesure où elle y introduisit, selon le témoignage des professeurs impliqués, de nouvelles pratiques d'enseignement des langues vivantes. Elle bénéficia également en France d'une certaine reconnaissance pédagogique à une époque d'ailleurs marquée par une expérimentation intense en didactique des langues étrangères. Elle servit à son tour de source d'inspiration à d'autres institutions culturelles françaises en Europe.

Toutefois, malgré son succès indéniable, la méthode orale fut abandonnée en Grèce après l'éloignement, en 1961, de Merlier et de son équipe enseignante de l'Institut. Optant pour un programme d'études "plus concentré» et pour une méthode, " susceptible de rendre [l'] enseignement [du français] plus rapidement efficace "1 la nouvelle direction traça sa propre politique enseignante et initia progressivement à l'Institut la méthode audio-visuelle. Mais même si, après quinze années de prédominance pédagogique incontestable, cette méthode d'enseignement disparut du paysage éducatif hellénique, elle réussit à relancer en Grèce un débat méthodologique 
et didactique sur le FLE qui devrait se poursuivre de manière beaucoup plus systématique au cours des décennies suivantes.

\section{BIBLIOGRAPHIE}

\section{Sources primaires}

Archives Diplomatiques de Nantes (ADN), archives de l'Institut français d'Athènes (AIFA), dossiers : 1058, 1068, 1070, 1071, 1072, 1074, 1090.

Centre d'Études d'Asie Mineure (CÉAM, Athènes), archives O. Merlier, dossier D5.

\section{Sources imprimées}

BURNEY, Pierre (1957). « La classe de conversation ». Les Cahiers pédagogiques pour l'enseignement du Second degré. Le Français Langue étrangère, 7, 31-32. EFTHIMIATOU-AlliSANDRATOU, Tassia (1957). « Enseignement oral intensif ». Les Cahiers pédagogiques pour l'enseignement du Second degré. Le Français Langue étrangère, 7, 61-62. GOUGENHEIM, Georges (1954). « Le Français Élémentaire ». The French Review, 27, 217-220. GOUGENHEIM, Georges (1955). « Le Français élémentaire. Étude sur une langue de base ». International Review of Education Internationale Zeitschrift für Erziehungswissenschaft / Revue Internationale de l'Éducation, 1, 401-412.

GOUGENHEIM, Georges (1957). « Efficacité et limitation dans l'enseignement ». Les Cahiers pédagogiques pour l'enseignement du Second degré, 7, 45-49.

GOUGENHEIM, Georges, MICHEA Réné, RIVENC Paul, SAUVAGEOT Aurélien. (1956). L'élaboration du français élémentaire : étude sur l'établissement d'un vocabulaire et d'une grammaire de base. Paris : Didier.

PAPANICOLAOU, Hélène, PAPASTAMATIOU, Hellé, TSOUCHLOU Irène, TZANETAKI Hellé (1957).

« Enseignement oral à l'Institut français d'Athènes ». Cahiers Pédagogiques pour l'enseignement du second degré, 7, 28-30.

VouTSINAS, Dimitri (1951). « Une méthode de travail vivante à l'Institut français d'Athènes ». Extrait des Amis de Sèvres, 9 et des Cahiers Pédagogiques, 1, sans pagination.

vouTSINAS, Dimitri (1953). «La méthode Octave Merlier pour l'enseignement du français ». Communication au $5^{\mathrm{e}}$ Congrès international des professeurs de langues vivantes, Sèvres, 7-10 avril 1953, sans pagination.

\section{Sources secondaires}

«Bibliographie concernant l'enseignement des langues vivantes - Unesco », Bibliographie préparée conjointement par la Fédération internationale des professeurs de langues vivantes, la 
Modern Language Association of America et l'Unesco http://unesdoc.unesco.org/images/ 0013/001337/133775fo.pdf [consulté le 14-05-2016].

CHEVALIER, Jean-Claude (2010). «Ferdinand Brunot et les débuts de l'École de préparation des professeurs de français à l'étranger ». Documents pour l'histoire du français langue étrangère ou seconde, 44, 15-27.

EFTHYMIOU, Loukia (2015). La formation des francisants en Grèce : 1836-1982. Paris : Publibook. PUREN, Christian (1988). Histoire des méthodologies de l'enseignement des langues. Paris : Nathan. RIONDET, Xavier (2013). « Les origines des Cahiers pédagogiques en 1945 ». Les Sciences de l'éducationPour l'Ère nouvelle, 46 : 113-136.

VÉRONIQUE, Georges Daniel (2010). « De l'ESPPPFE à l'UER EFPE : l'émergence d'un acteur universitaire de la didactique du FLE (1945-1980) ». Documents pour l'histoire du français langue étrangère ou seconde, 44, 89-102.

\section{NOTES}

1. Conférence présentée lors d'un stage portant sur la culture par les langues étrangères, organisée à Sèvres par l'École supérieure de préparation et de perfectionnement des professeurs de français à l'étranger (ESPPPFE). Cf. «Bibliographie concernant l'enseignement des langues vivantes - Unesco ». Sur Pierre Clarac, voir Véronique (2010:90).

2. Sur la méthode directe et les difficultés de son applicaton en France, notamment en second cycle, voir Puren (1988: 128-131).

1. ADN, AIFA, 1090 : Rapporteur Smyrnaki, «Exposé sur l'enseignement du cycle élémentaire », Institut Français d'Athènes, 5e Congrès de l'enseignement du français, Pâques 1955 (19-21 avril) : 14, 15. Sur la méthodologie traditionnelle, voir Puren (1988: 22-89); cf. « Allocution inaugurale de M. Merlier ", Institut Français d'Athènes, 5e Congrès de l'enseignement du français, Pâques 1955 (19-21 avril) : 5-6.

2. ADN, AIFA, 1072: Rapport sur l'activité de l'Institut français d'Athènes pendant l'année 1959-1960, fasc. I: 50.Sur les débuts de la constitution d'un «champ » du français langue étrangère en France, voir Chevalier (2010 : 15-27).

3. ADN, AIFA, 1090 : R. Milliex, "L'activité de l'Institut dans le passé, aujourd'hui et à l'avenir », Institut Français d'Athènes, Congrès de l'enseignement du français, Pâques 1948 (23-27 avril) : 4.

4. Cf. CÉAM, archives O. Merlier, dossier D5 : «Institut français d'Athènes de 1907 à 1952 » : 14.

1. ADN, AIFA, 1070 : Rapport sur l'activité de l'Institut français d'Athènes pour l'année 1946-1947 :

9.

2. Le versant grec de l'histoire de la méthode directe reste encore à écrire.

3. Cf. ADN, AIFA, 1070: Rapport sur l'activité de l'Institut français d'Athènes pour l'année 1951-1952: 32.

4. ADN, AIFA, 1072 : fasc. II : Rapport sur l'activité de l'Institut français d'Athènes pour l'année 1959-1960 : 58.

5. ADN, AIFA, 1055, « Note sur l'enseignement du français aux enfants de moins de 10 ans, et sur le rajeunissement des méthodes d'enseignement du français », 25 mai 1955.

6. ADN, AIFA, 1070 : Rapport sur l'activité de l'Institut français d'Athènes pour l'année 1946-1947 : 9. En 1948, les réponses des professeurs de l'Institut au questionnaire préparé par G. Mourelos révèlent leur adhésion à la méthode orale. Ibid., 1090: Georges Mourelos, «Rapport sur l'enseignement oral », IFA, Congrès de l'enseignement du français, Pâques 1948 (23-27 avril) : 51-56. 
7. ADN, AIFA, 1072 : « Rapport de Madame Papastamatiou sur les stages pédagogiques au Cours Spécial pendant l'année universitaire 1958-1959», Rapport sur l'activité de l'Institut français d'Athènes pour l'année 1958-1959, fasc. II : 17.

8. ADN, AIFA, 1070 : Rapport sur l'activité de l'Institut français d'Athènes pour l'année 1949-1950 : 36.

9. ADN, AIFA, 1068 : «La préparation au professorat à l'Institut français d'Athènes. Note établie par M. Milliex », 18-02-1956: 17.

10. ADN, AIFA, 1090: Agathonos, Capella, Pappa, Theodoridou, «Le Cours Spécial et notre préparation au métier de professeur de français ", Institut Français d'Athènes, Congrès de l'enseignement du français, Pâques 1950 (1-4 avril) : 142 ; ibid.,1072 : «Rapport de Mlle Leroux, conversation ", Rapport sur l'activité de l'Institut français d'Athènes pour l'année 1958-1959: 11-12.

11. ADN, AIFA, 1068 : Roger Milliex, « Note sur le Cours Spécial de Préparation au Professorat de Français ", juillet 1952 ; ibid, 1074 : Rapport de commission sur l'Institut français d'Athènes, signé Descotes, 30-06-1961 :48 ; cf. ibid., 1072, fasc. II : «Rapport de Madame Papastamatiou sur la formation et les stages pédagogiques au Cours Spécial pendant l'année universitaire 1959-1960 », Rapport sur l'activité de l'Institut français d'Athènes pour l'année 1959-1960 : 56.

12. ADN, AIFA, 1071: Rapport sur l'activité de l'Institut français d'Athènes pour l'année 1956-1957, fasc. II : 36.

13. ADN, AIFA, 1072: Rapport sur l'activité de l'Institut français d'Athènes pendant l'année 1959-1960, doc. cité : 50 ; ibid., dossier 1090 : R. Milliex, «L'activité de l'Institut dans le passé, aujourd'hui et à l'avenir ", doc. cité $: 11$.

1. ADN, AIFA, 1090: G. Mourelos: «Rapport sur l'enseignement oral», I. Tsouchlou, «L'enseignement au cours Préparatoire», H. Papanicolaou, «L'enseignement au cours élémentaire ", H. Mourelou, «L'enseignement par la chanson », IFA, Congrès de l'enseignement du français, Pâques 1948 (23-27 avril); Mlle Mavromati, «Présentation d'une leçon au Cours Oral », Madame Sangrioti, "Présentation d'une leçon au Cours Préparatoire», I. Tsouchlou, «Présentation d'une leçon au Cours Élémentaire », H. Papastamatiou, « Présentation d'une leçon au Cours Élémentaire», G. Mourelos, «Les principes de l'éducation nouvelle et notre enseignement ", IFA, Congrès de l'enseignement du français, Pâques 1950 (1-4 avril) ; Madame Smyrnaki, «Exposé sur l'enseignement du cycle élémentaire », IFA, 5e Congrès de l'enseignement du français, Pâques 1955 (19-21 avril).

2. ADN, AIFA, 1070 : Rapport sur l'activité de l'Institut français d'Athènes pour l'année 1948-1949 : 41-43; ibid.1090 : O. Merlier, «L'étude du français dans l'Enseignement Secondaire en Grèce » et "Allocution de Monsieur Zobanakis, Secrétaire Général du Ministère de l'Instruction Publique », IFA, Congrès de l'enseignement du français, Pâques 1950 (1-4 avril), p. 150-166, 185.

3. Ibid., 1058, Baulier: lettre $n^{\circ} 10857,15$ décembre 1952 signée Baulier; lettre $n^{\circ} 10744$, 20-01-1953, signée Milliex.

4. ADN, AIFA, 1063, H. Papanicolaou, I. Tsouchlou, H. Tzanetaki, « Considérations Générales sur le travail des Écoles annexes d'Athènes et de province pendant l'année scolaire 1957-1958 ", Rapport général d'Activité, IFA, $1958: 118$.

5. Autour de Sèvres se constituèrent plusieurs réseaux qui prolongeaient les rencontres entre enseignants français et étrangers. Les "Amis de Sèvres » en fut un, l'Association Nationale des Éducateurs des Classes Nouvelles de l'Enseignement du Second degré en lien direct avec les Cahiers pédagogiques en fut un autre. Sur l'histoire de la revue, voir Riondet 2013 : 113-136.

6. ADN, AIFA, 1048, « Note sur l'enseignement du français aux enfants de moins de 10 ans, et sur le rajeunissement des méthodes d'enseignement du français », 25 mai 1955.

7. Sur le français élémentaire, voir Gougenheim, Michéa, Rivenc, Sauvageot 1956; Gougenheim 1954 : 217-220 ; Gougenheim 1955 : 401-412 ; Gougenheim 1957 :45-49. 
8. ADN, AIFA, 1070 : Rapport sur l'activité de l'Institut français d'Athènes pendant l'année $1947-1948: 27$

9. ADN, AIFA, 1090 : Georges Mourelos, « Rapport sur l'enseignement oral », doc. cité : 44, 57-58.

1. ADN, AIFA, 1074 : Rapport de commission sur l'Institut français d'Athènes, doc. cité : 18, 21, 27, 44.

\section{RÉSUMÉS}

En 1946, des enseignants de l'Institut français d'Athènes, saisis par l'inefficacité de la méthodologie traditionnelle employée en Grèce pour l'enseignement du français, mirent au point, avec l'appui du directeur de l'établissement, une stratégie d'apprentissage " adaptée » aux besoins des débutants : la «méthode orale » inspirée de la méthodologie directe. L'application de la nouvelle méthode nécessita la préparation d'un matériel d'appoint, ainsi que l'organisation de stages pédagogiques et de perfectionnement à l'intention des maîtres de français en herbe et en exercice. Grâce à des stratégies efficaces de diffusion, la « méthode orale » bénéficia en Grèce et en France d'une reconnaissance pédagogique certaine et servit à son tour de source d'inspiration à d'autres institutions culturelles françaises en Europe. Bel exemple à la fois de transfert d'une pratique enseignante et d'ajustement de ses procédés et supports pédagogiques au sein de différentes institutions d'accueil, elle finit par disparaître du paysage éducatif hellénique en 1961.

In reaction to the ineffectiveness of the traditional methodology used in Greece for the teaching of the French language, a group of teachers of the French Institute of Athens developed in 1946 a new learning strategy more suitable for the needs of the beginners : the so called "oral method" had been inspired from the direct method. The implementation of the new method required the development/production of materials, as well as the organization of pedagogical courses for aspiring and confirmed teachers of French. Thanks to the use of effective strategies of diffusion, the "oral method" was acknowledged as a valid pedagogical method and became a source of inspiration for other cultural institutions in Europe. It ceased to be used in Greece in 1961.

\section{INDEX}

Mots-clés : méthode orale, méthode directe, enseignement du français, Institut français d'Athènes, lycée français de Vienne, Octave Merlier, Grèce, France

Keywords : oral method, direct method, teaching French, French Institute of Athens, French Lycee of Vienna, Octave Merlier, Greece, France

\section{AUTEURS}

\section{LOUKIA EFTHYMIOU}

Université nationale et

capodistrienne d'Athènes 
NICOLAS MANITAKIS

Université nationale et

capodistrienne d'Athènes 